\title{
A Study of Nucleotide Binding Domain of Glycerol Kinase Gene from Aloe
} vera

\author{
Sharma Suvarna ${ }^{1 *}$ and K Sharon ${ }^{2}$ \\ ${ }^{1}$ Department of Life Sciences, Kishinchand Chellaram College, D.W.Road, Churchgate, Mumbai, India \\ ${ }^{2}$ Department of Life Sciences, Kishinchand Chellaram College, D.W.Road, Churchgate, Mumbai, India \\ *Corresponding author: suvarsharma@rediffmail.com, Mobile: 9869525362
}

\section{Available online at: www.isroset.org}

Received: 30/Dec/2018, Accepted: 23/Jan/2019, Online: 28/Feb/2019

\begin{abstract}
Aloe vera, belonging to family Liliaceae, is a monocot and used also for medicinal purpose. They are succulent and retain large amount of water in their leaves. In the recent decade few genes from Aloe vera have been elucidated, some being stress inducible. Glycerol kinase is the enzyme involved in the first step of Glycerol metabolism. In order to study the gene, its nucleotide binding domain was targeted by PCR approach. A 256bp long amplification product was obtained which on sequencing and in- silico analysis confirmed to be NBD domain of Glycerol kinase. This domain belongs to the superfamily NBD_Sugar kinase_HSP70_Actin, which all harbor the same conserved domain. It shows 52\% alignment with the FGGY CTerminal region of Escherichia coli, which also harbor sites for ATP binding. This paper confirms the presence and studies the structure of the NBD domain, thereby indicating the presence of a glycerol kinase gene in Aloe vera genome for the first time.
\end{abstract}

Keywords: NBD, Hsp70, Actin, PCR, FGGY, ATP

\section{INTRODUCTION}

Plants respond in a myriad of ways to stress, which can be triggered by abiotic or biotic factors. The response may be in the form of production of heat shock proteins, closure of stomata aperture, activation of stress induced genes etc. One such mechanism is where a eukaryotic cell is likely to maintain the osmotic potential of the cytoplasm by increased assemblage of glycerol [1,2]. It is conceivably due to production of glycerol in large quantities or prevention of glycerol catabolism. The latter is feasible considering loss of function mutation in the enzymes of catabolic pathway, as opposed to devoting energy in generating excess.

Glycerol is utilized by the cells as a source of carbon, and it is also a breakdown product of glyceraldehyde. Glycerol is broken down to glycerol-3- phosphate (G3P) by Glycerol kinase and G3P is further involved production of fatty acids as observed in the pathway Figure $1[3,4 \& 5]$. Accumulation of glycerol in the cells can thus be possible if glycerol kinase is non- functional. 


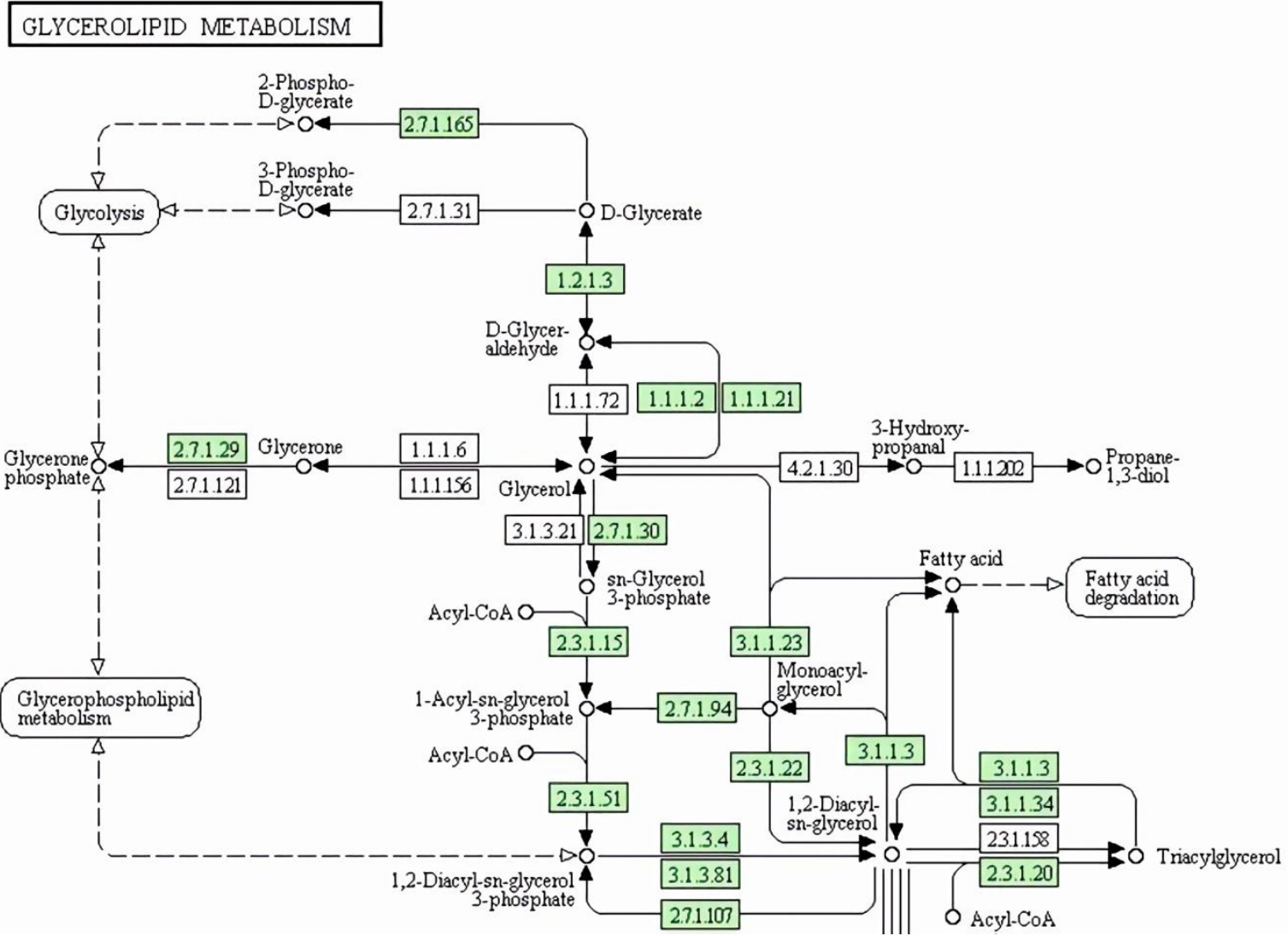

Figure 1: Metabolic pathway of Glycerolipid in Saccharomyces cereviseae (Ref: Kyoto Encyclopedia of Genes and Genomes [3, 4 \&5]

Glycerol kinase is recognized by the EC number 2.7.1.30. It is listed under 'Transferases' class of enzymes [6]. It converts glycerol to glycerol-3- phosphate by utilizing ATP. Glycerol kinase enzyme is made up of 506 amino acids and harbours two major domains- FGGY_N and FGGY-C terminal domains. The $\mathrm{N}$ terminal domain is involved in substrate binding whereas the $\mathrm{C}$ terminal is associated with nucleotide binding. The $\mathrm{C}$ terminal domain possesses four ATP binding sites, especially a motif that binds to ATP between $400-450^{\text {th }}$ amino acid (PDB ID 034153) [7].

Aloe vera is a monocot and a succulent plant. Retention of water aids them in surviving abiotic stress factors such as high temperature, drought and high salinity. This property of Aloe vera makes them an ideal plant for studying stress induced molecules and genes. Stress inducible genes such as DREB [8] and HVA22 [9] genes have been isolated from Aloe vera. The current work focuses on studying Glycerol kinase gene, targeting its FGGY_C terminal domain by PCR. Glycerol kinase gene in Aloe vera is being reported for the first time.

Section I contains the introduction of stress resistant mechanisms in plants, discussing in brief the glycerol assimilation mechanism and concluding by discussing the strategy applied to target the gene for glycerol kinase in Aloe vera. Section II discusses the methodology of PCR, Sequencing and In silico analysis of the data. Section III deals with the results of the above mentioned methodology with images and figures. Section IV is the Discussion which correlates the FGGY_N terminal sequence of Aloe vera Glycerol kinase gene with the literature available. Finally Section V concludes the study by mentioning the possible methods of continuing the research.

\section{MATERIALS AND METHODS}

\section{Genomic DNA extraction and PCR}

Four grams of young leaf of Aloe vera plant were used for genomic DNA extraction. CTAB technique with modification (Instead of liquid nitrogen, fresh leaves were directly crushed in buffer) was used to extract High molecular weight genomic DNA from Aloe vera leaf. DNA was analyzed by Agarose Gel electrophoresis for degradation \& estimation was carried out using UV spectrophotometric analysis (Thermo Electron Corp.) at $260 \mathrm{~nm} \& 280 \mathrm{~nm}$. Primers for the PCR were designed for Glycerol Kinase using Primer- BLAST program from NCBI, 
and the primers were (g FP)5'ATATATGGATCCTTGAAAAACTGTGGAGCC 3' \& (g_RP)5' ATGAACAGAAGCACCTGTAG 3'; they were synthesized by Sigma- Aldrich Pvt. Ltd. The genomic DNA sample was purified by PCR Purelink Purification Kit (K-310001). For PCR, purified genomic DNA as template, g_FP and g_RP primers and PCR Reaction Mix (Sigma's Red Taq PCR supermix) were used for amplification of the glycerol kinase homolog using ABS Thermal Cycler 2720. AGE was performed using low range DNA molecular ladder for reference.

\section{Sequencing:}

PCR products obtained with genomic DNA and g_FP\& g_RP primer was sent for bi- directional sequencing to Chromous Biotech Pvt Ltd. Sanger's Dideoxy technique of sequencing was performed.

\section{Insilico analysis:}

DNA sequence of the PCR amplification Product obtained was subjected to NCBI BLAST (Sense \& Antisense strands) [10]. A BLASTn, BLASTx [11] and CDD (Conserved Domain Database) search was performed [12]. ORF was found using NCBI's ORF finder program. The potential exons present within the fragment were analysed using FGENESH 2.0 version Softberry Program [13]. Protein prediction was performed at Predict Protein website [14]. The predicted protein sequence was searched against PDB database.

\section{RESULTS}

\section{Genomic DNA results:}

A single high molecular weight band close to the well was observed on AGE (Figure 2); when subjected to UV spectrophotometric analysis it was found to be $0.5 \mathrm{microgram} / \mathrm{microlt}$.

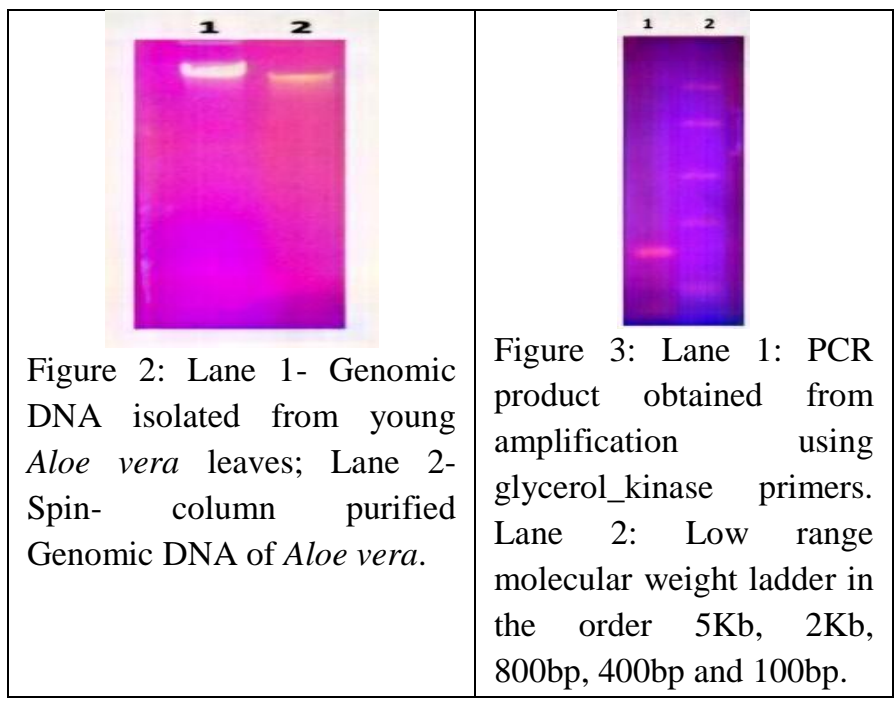

\section{PCR Results:}

A single band positioned between 400bp and 100bp was observed as shown in Figure 3.

\section{Sequencing \& Bioinformatics Analysis:}

Sequencing of this PCR product revealed an approximately 250bp long DNA. BLASTn analysis of this sequence showed $98 \%$ identity with glycerol kinase gene from Gluconobacter oxydans. BLASTx results of this sequence showed highest identity (98\%) with glycerol kinase protein from Gluconobacter oxydans with a score of 124 and query coverage of $96 \%$. Maximum hits were observed in the range of $80-200$ on the scorecard and showing identity with glycerol kinase gene from different organisms such as Sphingomonas sps, Acetobacter nitrogenifigens, Caulobacter sp., etc. The percentage identity ranged between $96 \%$ to $55 \%$; query coverage ranged between $93 \%$ and $74 \%$. The CDD search revealed that this fragment harbored an NBD_sugar-kinase_HSP70_actin domain superfamily. It shows a complete ORF in the +1 Reading frame. Protein modeling of the translated sequence resulted in many hits for Glycerol Kinase and Heat shock cognate $71 \mathrm{kDa}$ protein. Figure $4 \mathrm{a}$ and Figure $4 \mathrm{~b}$ shows the position of the nucleotide binding domain in a typical Glycerol kinase or Heat shock protein.

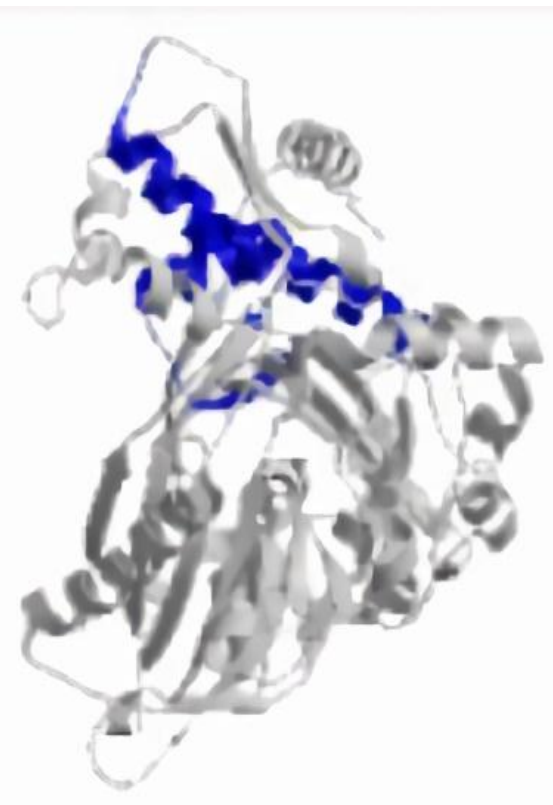

a. Swiss Model Results: Protein Glycerol Kinase, X- ray Diffraction $3.05 \mathrm{~A}$ 


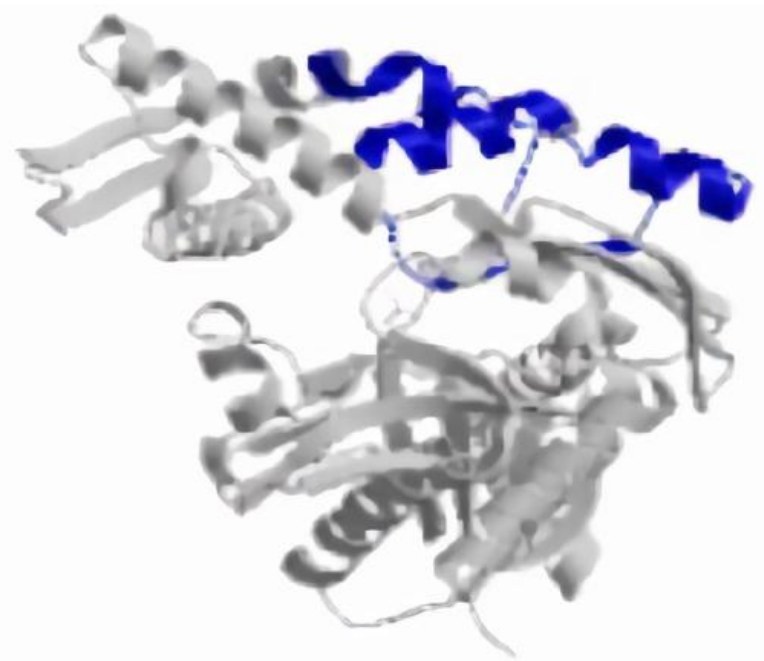

b. Swiss Model Results: Heat Shock cognate $71 \mathrm{kDa}$ protein, X-ray diffraction, $1.94 \mathrm{~A}$

Figure 4: The swiss model results for a. glycerol kinase and b. Heat shock protein. The blue shaded region represents the respective domain in the nucleotide binding domain [15]

The structure for the predicted protein sequence was built using Swiss modeling software. The structure showed $50 \%$ sequence identity with Glycerol kinase protein sequence. The structure predicted is as shown in Figure 5.
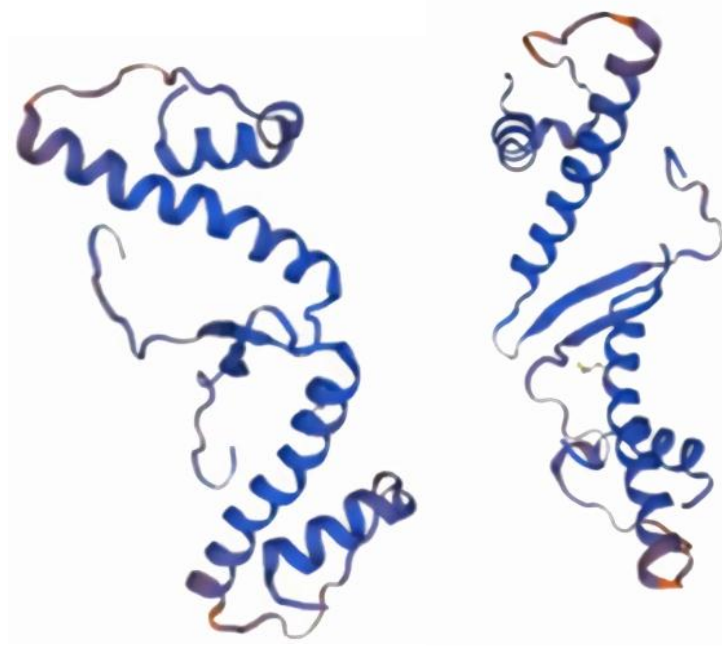

Figure 5: The predicted model of glycerol kinase protein from Aloe vera $[16,17]$

The predicted protein sequence was searched against Protein Data Bank Database. The results showed 52\% identity, Scoring 84.344bits and zero gaps with Glycerol kinase gene of Escherichia coli. The insilico translated protein sequence aligned with $352^{\text {nd }}$ amino acid to $426^{\text {th }}$ amino acid of the glycerol kinase gene (Figure 6). Figure 7 shows the Glycerol kinase protein structure in Homo sapiens, in which the motif arrangements remain same as in Escherichia coli.

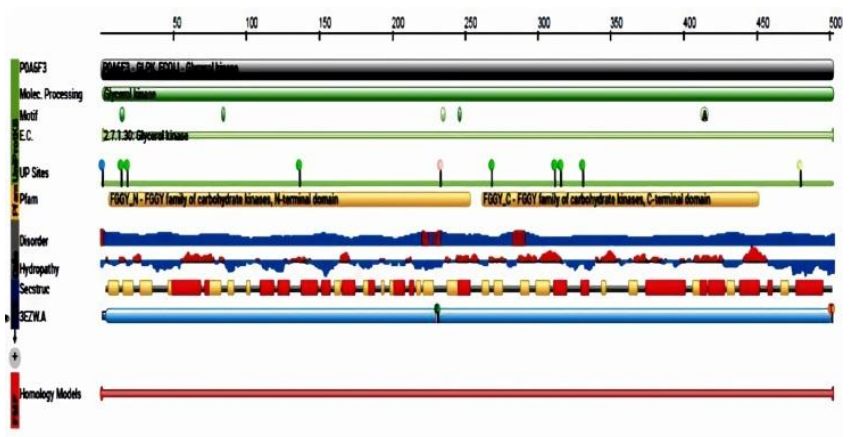

Figure 6: The structure of glycerol kinase gene studied in Escherichia coli as procured from PDB P0A6F3 (GLPK_ECOLI) [18]

Organism : Homo sapiens (human)

Gene: $\quad 2710$

Definition : K00864 glycerol kinase [EC:2.7.1.30] | (RefSeq) GK, GK1, GKD; glycerol kinase

\begin{tabular}{|l|l|l|l|l|}
\hline Motif id & From & To & Definition & Evalue Score \\
\hline
\end{tabular}

\begin{tabular}{|l|l|l|l|l|l|}
\hline \hline pf:FGGY_N 13 & 272 & FGGY family of carbohydrate kinases, N-terminal domain & $1.7 e-96$ & - \\
\hline
\end{tabular}

\begin{tabular}{|l|l|l|l|l|l|l|}
\hline \hline pf:FGGY_C & 281 & 472 & FGGY family of carbohydrate kinases, C-terminal domain & $2.5 e-64$ & - \\
\hline
\end{tabular}

Search GENES with the same motifs

hsa:2710

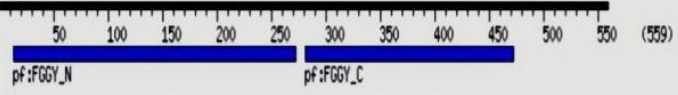

Figure 7: The structure of glycerol kinase gene studied in Homo sapiens as procured from KEGG [3, 4 \& 5]

\section{DISCUSSION}

The glycerol kinase protein encoded by this gene GK belongs to the FGGY kinase family. It is the key enzyme in the regulation of glycerol uptake and metabolism. As mutations in the metabolic enzymes is related to breakdown of glycerol in human results in diseases and syndromes which lead to death, it may be conceivable that glycerol or G3P might also participate in disease physiology of plants and this role is yet to be discovered [19]. In such a study conducted, the following observation and hypothesis was made: A pathogenic fungi relies on glycerol for its growth and establishment, even in the presence of sucrose or glucose, from plants; it is these pathogen- mediated changes 
in glycerol levels and its utilization that signals the plant to initiate defense [19].

A gli1 glycerol insensitive mutant Arabidopsis seed was isolated which was unable to utilize glycerol and hence transiently accumulated them; but they were found to be more resistant to hyperosmotic stress, salt stress, oxidative stress, freezing and desiccation [20]. Arabidopsis mutants for gli1, act1 and transgenic GDP1- Arabidopsis plants showed gradual increase of glycerol levels with increase in salt stress; it was hypothesized that glycerol retention/ efflux in the cell are affected at $200 \mathrm{mM} \mathrm{NaCl}$ [21].

From figure 6, we can observe that the segment of gene isolated in Aloe vera aligns with the FGGY_C terminal domain of Glycerol kinase gene from Escherichia coli. The green dots signify the ATP binding sites in the $\mathrm{C}$ terminal. Especially in the region isolated there seems to be a nucleotide phosphate binding site- ATP binding motif. This further confirms the presence of NBD- Nucleotide binding domain in the isolated gene belongs to sugar kinase hsp70_actin superfamily. Although the Aloe vera glycerol kinase partial gene shows identity with that from a prokaryote, Figure 7 confirms that the domain remains same across prokaryotes and eukaryotes. The FGGY_N and FGGY_C domains in both organisms from different kingdoms are present in the similar location.

The partial sequence was submitted to NCBI via Bankit under the accession ID KT273416. The information of the partial GK gene sequence obtained from this study can be used to determine the complete coding sequence of GK and its protein isolation. From the literature it was observed that the system having mutated GK gene showed resistance to pathogen attack and many types of abiotic stresses.

\section{CONCLUSION}

A partial GK gene sequence from Aloe vera genomic DNA was isolated and it's predicted protein structure was studied in silico. The domain isolated from Aloe vera genome can be observed for mutations by expression of same using total RNA from Aloe vera and subjecting it to coding sequence (gk) specific primers and deduce it's complete coding sequences. Simultaneously, the Glycerol kinase protein can be isolated. By correlating the Glycerol kinase protein content in the plant under stress conditions, it could possible to find any role of glycerol kinase in imparting stress resistance in Aloe vera.

\section{Funding:}

This project was funded for by University Grants Commission, Delhi as a UGC Major Project.

\section{Acknowledgement:}

We would like to thank K C College and UGC.

\section{Bibliography:}

[1]. Bo Shen, Stefan Hohmann, Richard G. Jensen, and Hans J. Bohnert, Roles of Sugar Alcohols in Osmotic Stress Adaptation. Replacement of Glycerol by Mannitol and Sorbitol in Yeast, Plant Physiology, Vol 121, pp: 45-52 (1999).

[2]. Afzal Husain, Dan Sato, Ghulam Jeelani, Tomoyoshi Soga, Tomoyoshi Nozaki, Dramatic Increase in Glycerol Biosynthesis upon Oxidative Stress in the Anaerobic Protozoan Parasite Entamoeba histolytica, PLOS: Neglected Tropical Disease, Vol 6(9) (2012)

[3]. Kanehisa, Furumichi, M., Tanabe, M., Sato, Y., and Morishima, K.; KEGG: new perspectives on genomes, pathways, diseases and drugs. Nucleic Acids Res. 45, D353-D361 (2017).

[4]. Kanehisa, M., Sato, Y., Kawashima, M., Furumichi, M., and Tanabe, M.; KEGG as a reference resource for gene and protein annotation. Nucleic Acids Res. 44, D457-D462 (2016).

[5]. Kanehisa, M. and Goto, S.; KEGG: Kyoto Encyclopedia of Genes and Genomes. Nucleic Acids Res. 28, 27-30 (2000).

[6]. Kanehisa, M.; Enzyme annotation and metabolic reconstruction using KEGG. Methods Mol. Biol. 1611, 135-145 (2017).

[7]. H.M. Berman, J. Westbrook, Z. Feng, G. Gilliland, T.N. Bhat, H. Weissig, I.N. Shindyalov, P.E. Bourne. The Protein Data Bank Nucleic Acids Research, 28: 235-242. (2000)

[8]. Wang, YM. \& He, CF. Isolation and Characterization of a ColdInduced DREB Gene from Aloe Vera L.Plant Mol Biol Rep 25: 121. (2007) https://doi.org/10.1007/s11105-007-0014-3

[9]. Kadirvelu Sharon and Sharma Suvarna, Cloning of HVA22 Homolog from Aloe vera and Preliminary Study of Transgenic Plant Development, International journal of pure and applied bioscience, Vol 5(6), pp: 1113-1121 (2017)

[10]. Altschul, S. G. Basic local alignment search tool. J. Mol. Biol., 215:403-410(1990)

[11]. Gish, W. \& States D.J. Identification of protein coding regions by database similarity search Nature Genet. , 3:266-272. (1993)

[12]. Bauer, M. CDD: a Conserved Domain Database for the functional annotation of proteins". Nucleic Acids Res., 39(D) 225-9 (2011).

[13]. Solovyev V, K. P. Automatic annotation of eukaryotic genes, pseudogenes and promoters. Genome Biol., 7, Suppl 1: P. 10.110.12 (2006).

[14]. Yachdav, G., Kloppmann, E., Kajan, L., Hecht, M., Goldberg, T., Hamp, T., et al. PredictProtein---an open resource for online prediction of protein structural and functional features. Nucleic acids research, gku366. (2014).

[15]. Bienert, S., Waterhouse, A., de Beer, T.A.P., Tauriello, G., Studer, G., Bordoli, L., Schwede, T. The SWISS-MODEL 
Repository - new features and functionality. Nucleic Acids Res. 45, D313-D319 (2017).

[16]. Waterhouse, A., Bertoni, M., Bienert, S., Studer, G., Tauriello, G., Gumienny, R., Heer, F.T., de Beer, T.A.P., Rempfer, C., Bordoli, L., Lepore, R., Schwede, T. SWISS-MODEL: homology modelling of protein structures and complexes. Nucleic Acids Res. 46(W1), W296-W303 (2018).

[17]. Guex, N., Peitsch, M.C., Schwede, T. Automated comparative protein structure modeling with SWISS-MODEL and SwissPdbViewer: A historical perspective. Electrophoresis 30, S162S173 (2009).

[18]. H.R. Drew, R.M. Wing, T. Takano, C. Broka, S. Tanaka, K. Itakura, R.E.Dickerson Structure of a B-DNA dodecamer: conformation and dynamics Proc.Natl.Acad.Sci.USA 78: 2179$2183(1981)$

[19]. Srivathsa C Venugopal, Bidisha Chanda, Lisa Vaillancourt, Aardra Kachroo, and Pradeep Kachroo The common metabolite glycerol-3-phosphate is a novel regulator of plant defense signaling. Plant Signaling and Behaviour, 4:8, $746-749$ (2009).

[20]. Eastmond Peter J Glycerol- insensitive Arabidopsis mutants: gli1 seedlings lack glycerol kinase, accumulate glycerol and are more resistant to abiotic stress. Plant J, 37(4): 617- 25. (2004).

[21]. Ahmed Bahieldin, Jamal S. M. Sabir, Ahmed Ramadan, Ahmed M. Alzohairy, Rania A. Younis, Ahmed M. Shokry, Nour O. Gadalla, Sherif Edris, Sabah M. Hassan, Magdy A. Al-Kordy, Khalid B. H. Kamal,Samar Rabah, Osama A. Abuzinadah and Fotouh M. El-Domyati Control of glycerol biosynthesis under high salt stress in Arabidopsis. Functional Plant Biology, 41(1) 87-95. (2013).

\section{Author's Profile:}

\section{Dr. Suvarna Sharma}

Dr. Suvarna Sharma is currently working as Assistant Professor in the Department of Life Sciences at K C College. She has Ph. D from Tata Memorial Hospital and M.Sc from TIFR. She was the Principal Investigator of UGC Major Project from 2012- 2015. She also headed the Molecular Biology division at

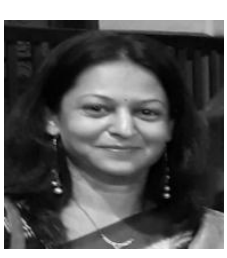

Metropolis. She has guided undergraduate projects under JigyaasaScience Honors Program at K C College. She has great interest in Plant molecular biology.

\section{Ms. Sharon K}

Ms. Sharon $\mathrm{K}$ is currently pursuing her $\mathrm{Ph} . \mathrm{D}$ in Life Sciences from K C College. She has previously served as Assistant Professor in Department of Biotechnology at K C College. She also worked as Junior Research Fellow for UGC Major Project at K C College. She has Master's degree in Biotechnology and a certificate in Biostatistics and Design of Experiments pursued from NPTEL- IIT Madras Online Portal.

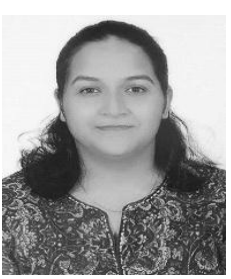

